\title{
Can a cardiorespiratory field parameter assess both cardiovascular and respiratory fitness in schoolchildren?
}

\author{
Louisa Ming Yan Chung*, Lina Pui Yu Chow, Eric Cheuk Kuen Tsang, Joanne Wai Yee Chung \\ Department of Health and Physical Education, The Hong Kong Institute of Education, Hong Kong, China; \\ *Corresponding Author: chungmy@ied.edu.hk
}

Received 31 October 2013; revised 28 November 2013; accepted 12 December 2013

Copyright (c) 2014 Louisa Ming Yan Chung et al. This is an open access article distributed under the Creative Commons Attribution License, which permits unrestricted use, distribution, and reproduction in any medium, provided the original work is properly cited. In accordance of the Creative Commons Attribution License all Copyrights (C) 2014 are reserved for SCIRP and the owner of the intellectual property Louisa Ming Yan Chung et al. All Copyright (C) 2014 are guarded by law and by SCIRP as a guardian.

\section{ABSTRACT}

Background: Cardiorespiratory fitness involves both cardiovascular and respiratory capacities. However, existing methods have been criticised for reflecting cardiovascular fitness, but not pulmonary fitness. The objective of this study was to investigate the relationship between these two testing parameters. Methods: A crosssectional population-based physical fitness assessment was conducted in 23 schools. The body height and weight, lung capacity, and step test results of students aged 10 - 18 were recorded. Criterion-referenced standards from the China Sports Bureau were adopted, as they include separate measurements for cardiovascular and pulmonary fitness. Results: The Pearson coefficients of lung capacity and the step test index from 13,028 schoolchildren ranged from 0.007 to 0.086 and from 0.026 to 0.105 for boys and girls, respectively, showing that poor correlations exist between the two parameters. Cluster analysis resulted in three clusters of children with similar characteristics. A good degree of similarity was found between the two parameters in children obtaining an "excellent" grade, but only a moderate degree of similarity between the two parameters in those obtaining a "good", "pass" or "fail" grade. Conclusion: When cardiovascular fitness and pulmonary fitness are considered separately, there is a poor correlation between them, thus indicating further studies of cardiopulmonary fitness measurements is necessary.

\section{KEYWORDS}

Cardiovascular Fitness; Pulmonary Fitness; Cardiorespiratory Fitness; Field Assessment; Schoolchildren

\section{INTRODUCTION}

Cardiorespiratory fitness (CRF) indicates a healthy cardiovascular profile in youth [1-3]. CRF assessments are common in physical education, exercise programmes and health-related physical fitness assessments. The literature reports that CRF is sometimes used instead of cardiovascular fitness to reflect the functional capabilities of the heart, blood vessels, lungs and skeletal muscles as a body system in performing large-muscle, dynamic, moderate to high-intensity exercises over relatively long periods [4]. Ortega et al. [2] define CRF as the overall capacity of the cardiovascular and respiratory systems and the ability to perform prolonged strenuous exercise. As CRF involves both the cardiovascular and pulmonary systems, it is reasonable from the perspective of human biology to integrate their functions as the circulation system. However, is it true that cardiovascular fitness equates to respiratory fitness? There is a gap in the research on the relationship between these two fitness parameters, and the aim of this study was thus to explore whether a single field test can assess both cardiovascular and respiratory fitness.

CRF assessments include maximal exercise testing, submaximal exercise testing and field testing. Maximal and submaximal testing are more accurate than field testing in tracking an individual's CRF function, but they require trained personnel, complex equipment and a long testing period, and pose a higher level of risk. Thus, they 
are infeasible in large group assessments. In practice, three types of CRF field tests are commonly applied: fixed distance tests, fixed time tests and bench stepping protocols. Fixed distance and fixed time tests usually require individuals to run at a maximal or near-maximal level of exertion for performance evaluation. Most studies of CRF field tests consider the 20-metre shuttle run [5-11] as the CRF field assessment item. However, caution must be exercised when using this method, particularly in assessing individuals at moderate to high risk of cardiovascular or musculoskeletal diseases, as they may suffer complications [4]. In addition, fixed distance and time tests also require space and mobility, which may raise concerns in field settings [12]. Bench stepping protocols, in contrast, require a relatively small space and less time in largescale field measurements. However, applications of bench stepping protocols such as the Harvard Step Test [13], YMCA Step Test [14] and China Sports Bureau step test [15] are rare in the literature.

The literature on field tests for lung capacity measurement is also sparse, although specific lung capacity tests have been performed in laboratory settings on patients with thoracic problems [16-18]. In addition, two studies have reported lung capacity field tests carried out in conjunction with population-based physical fitness assessments conducted using the protocols of the China Sports Bureau [19,20]. Cardiovascular fitness and cardiopulmonary fitness are commonly considered interchangeable in assessing CRF [4]. The current study queries whether a field assessment of cardiovascular fitness is a good indicator of pulmonary fitness, and examines the correlation between the assessment results of these two physiological systems.

\section{MATERIALS AND METHODS}

\subsection{Participants}

Primary and secondary schoolchildren in Hong Kong were invited to participate in a large-scale physical fitness assessment from September 2005 to December 2011. A snowball sampling process was adopted to recruit schools for participation and to refer additional schools to the research team. In carrying out the study, which was approved by the university's ethics committee, the researchers followed the principles of the Helsinki Declaration of 1975. All of the participating children and their parents were given information sheets describing the purpose and methods of the assessment procedures and the application of the physical fitness results. They were asked to sign consent forms before the assessments were administered. Each participating student completed a battery of field tests according to the China Education Bureau Protocol [15].

\subsection{Design}

The physical fitness assessment was a cross-sectional survey. This study constitutes secondary analysis of the data collected in that assessment [21].

\subsection{Instruments}

The equipment used to assess lung capacity was an electronic sensor measuring the volume of outflow air in millilitres. Participants were instructed to breathe in with as much expanded lung capacity as possible, and then breathe out as much air as possible through the mouth piece of the sensor. Six pieces of equipment of the same model were used in the participating schools, and they were calibrated each day using a laboratory-certified syringe before data collection.

A step test was used to assess participants' cardiovascular fitness. Two students were tested simultaneously using a long, 25-centimetre-high step box. They were instructed to step on and off the box repeatedly, alternating their right and left feet. Students were motivated by and encouraged to follow music with a programmed rhythm for three minutes. This protocol was intended to standardise the type, intensity and time of the aerobic exercise prior to the assessment. After three minutes of exercise, the students were asked to rest in a sitting position, and a trained researcher measured their pulse rates at the 60 - 75th, 120 - 135th and 180 - 195th second after completing the test. A step test index was then calculated [step test index $=$ (total stepping time in seconds $\times 100) /$ ( $4 \times$ (SUM of three measured pulse rates)] to determine whether participants' heartbeats recovered quickly from a vigorous to resting level.

\subsection{Procedure}

Physical education teachers and university student helpers were trained to take field measurements of the students' body height and weight, lung capacity and poststep test pulse rate at the 6 primary and 17 secondary schools that agreed to participate in the physical fitness assessments. Children under the age of 10 were not asked to take the lung capacity test or step test, and some of the children were not of Chinese ethnicity. The final sample included 13,028 children aged 10 to 18 .

Criterion-referenced standards for Chinese schoolchildren [15] were used to classify the results of the lung capacity and step tests as “excellent”, “good”, “pass” and "fail". The volume measurements recorded in the lung capacity test were transformed into a lung capacity index by dividing lung capacity in millilitres by body weight. The corresponding lung capacity indexscores for the four aforementioned standards were 63 or above, 53 - 62, 29 45 and 28 or below for boys and 54 or above, 38 - 53, 18 - 37 and 17 or below for girls. The step test index 
scores for the four standards were 64 or above, 51 - 63, 41 - 50 and 40 or below for boys and 62 or above, 48 61, 40 - 47 and 39 or below for girls.

\subsection{Statistical Analysis}

All analysis was conducted using the Statistical Package for the Social Sciences, version 20. The results are stratified by age and sex, and stated by the mean, standard deviation, 95\% confidence interval (CI) of the lung capacity and step test indices, and the percentiles of 5th, 10th, 25th, 50th, 75th, 90th and 95th. The Pearson product-moment correlation coefficients of lung capacity and the step test index were computed to reveal the relationship between the pulmonary fitness parameter measured as lung capacity and the cardiovascular parameter measured as the step test index.

Hierarchical cluster analysis with squared Euclidean distance was performed to categorise the participants into meaningful groups by age, lung capacity and step test index by maximising the similarity of those within each cluster while maximising the dissimilarity between groups. Chi-squared test was used to measure the effect size for gender and lung capacity test ranks and step test ranks by computed clusters. Since there were four ranks in each test, Cramer's V statistics were used. According to Cohen (1988), $d=0.10, d=0.30$ and $d=0.50$ were used for the effect sizes of small, medium and large respectively.

\section{RESULTS}

Of the 13,028 participants, 7154 (54.9\%) were boys and 5874 (45.1\%) girls. The mean and standard deviation of age for the boys were 13.9 and 1.87 , respectively, and those for the girls were 13.9 and 1.88 , respectively. Some of the children were unable to complete the step test or lung capacity test, with complete test data obtained for 9389 participants.

\subsection{Normative Standard of Testing Results}

Table 1 presented the normative standards of the lung capacity and step tests by sex. In general, lung capacity performance was found to increase with age for both boys and girls. Amongst boys, the variances in the lung capacity results between the ages of 13 and 18 were greater than those at a younger age, whereas the variances for girls remained relatively constant, except for an unusual difference in the 95\% C.I. found at age 13. However, step test performance showed a decreasing trend from age 12 to 17 in boys and a significant decreasing trend in girls from age 12 . The variances in the step test index results were similar for boys, but larger variances were found for girls aged 12 to 13 .

\subsection{Correlations of Testing Results}

The Pearson-moment coefficients for the lung capacity results in millilitres and the step test index scores are listed as $R^{2}$ in Figures 1 and 2. The correlation coefficients range from 0.007 to 0.086 for boys, with a significant correlation found at age 12. Those for girls range from 0.026 to 0.105 , with a significant correlation found at ages 13 and 14. Scatterplots of lung capacity and the step test index by sex and age are shown in Figures 1(a) to $(\mathrm{r})$. The best fit lines are interpolated to illustrate the correlations.

\subsection{Cluster Analysis}

Taking sex, age, lung capacity in millilitres and the step test index as cluster variables and three as the number of defined solutions, the children were categorised into three clusters with the shortest distances between characteristics: $61.8 \%(\mathrm{n}=5828), 36.7 \%(\mathrm{n}=3466)$ and $1.5 \%(\mathrm{n}=139)$ in Clusters A, B and C, respectively. In Cluster $\mathrm{A}$, the number of boys achieving excellent lung capacity results is similar to the number achieving excellent step test results at all testing ages. More boys achieved good, pass and fail results in the lung capacity test than in the step test. Similar numbers of girls achieved excellent and good results in the lung capacity and step tests, whilst more girls achieved pass and fair lung capacity results than step test results. The effect sizes for both lung capacity ranks $(\mathrm{d}=0.19)$ and step tests $(\mathrm{d}=0.068)$ were small in cluster $\mathrm{A}$.

In Cluster B, the number of boys with excellent lung capacity results was similar to the number with excellent step test results, whereas fewer boys had good and pass lung capacity results relative to step test results. Fewer boys failed the lung capacity test than the step test. Similar numbers of girls achieved excellent lung capacity and step test results, although the number of good and pass results was far less for lung capacity than for the step test. None of the girls in Cluster B failed the lung capacity test, although some failed the step test. The effect sizes for both lung capacity ranks $(d=0.146)$ and step tests $(d=0.099)$ were small in cluster B. Finally, in Cluster $\mathrm{C}$, a similar number of boys achieved excellent results in the lung capacity and step test, although fewer boys achieved a good or pass in the lung capacity test than in the step test. No boys in this cluster failed the lung capacity test, but several 13- and 15-year-old boys failed the step test. Similar numbers of girls in Cluster $\mathrm{C}$ achieved excellent lung capacity and step test results. All girls in Cluster $\mathrm{C}$ scored excellent in lung capacity, whereas some achieved a good or pass in the step test. No girl failed the step test. The effect sizes for both lung capacity ranks $(d=0.059)$ and step tests $(d=0.081)$ were small in cluster $\mathrm{C}$. 
Tab le 1. Normative data from lung capacity test and step test.

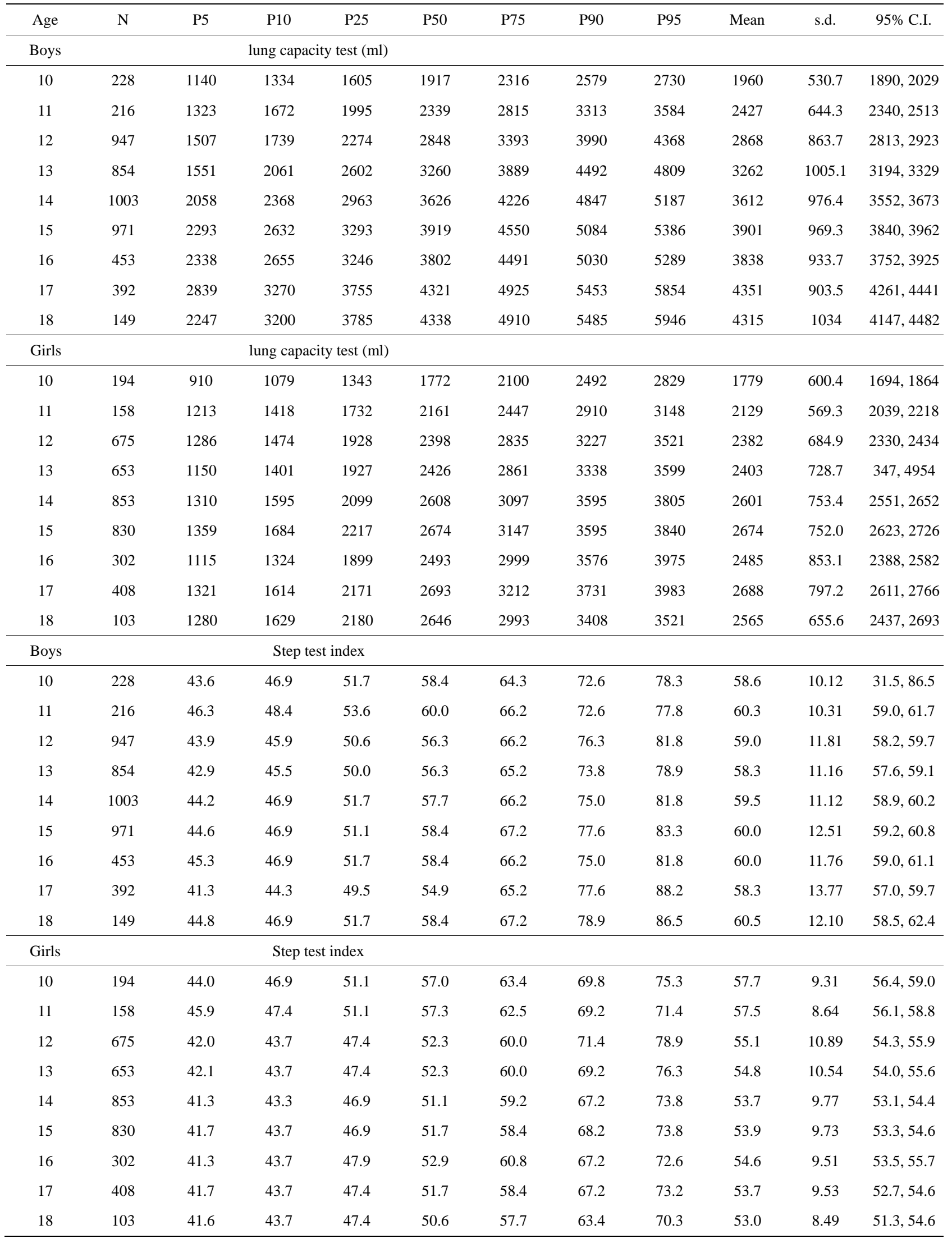




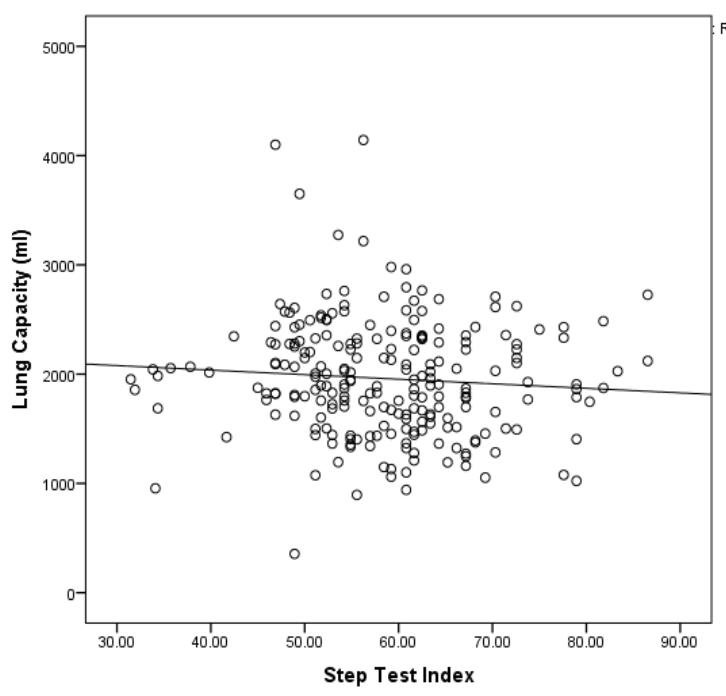

(a) 10-year-old boys

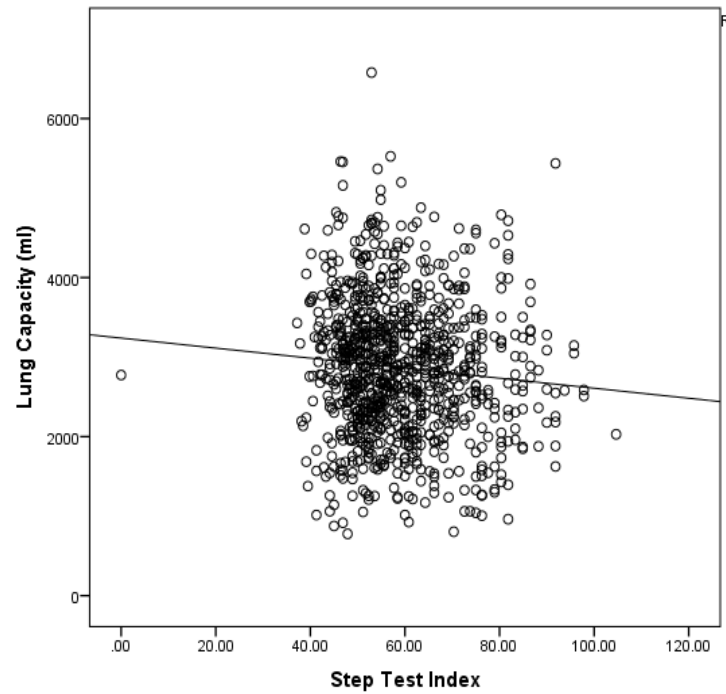

(c) 12-year-old boys

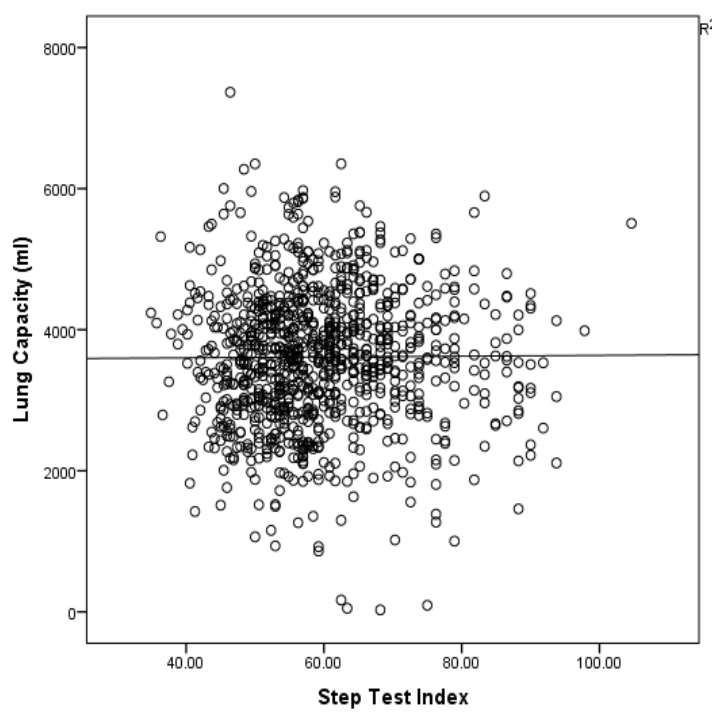

(e) 14-year-old boys

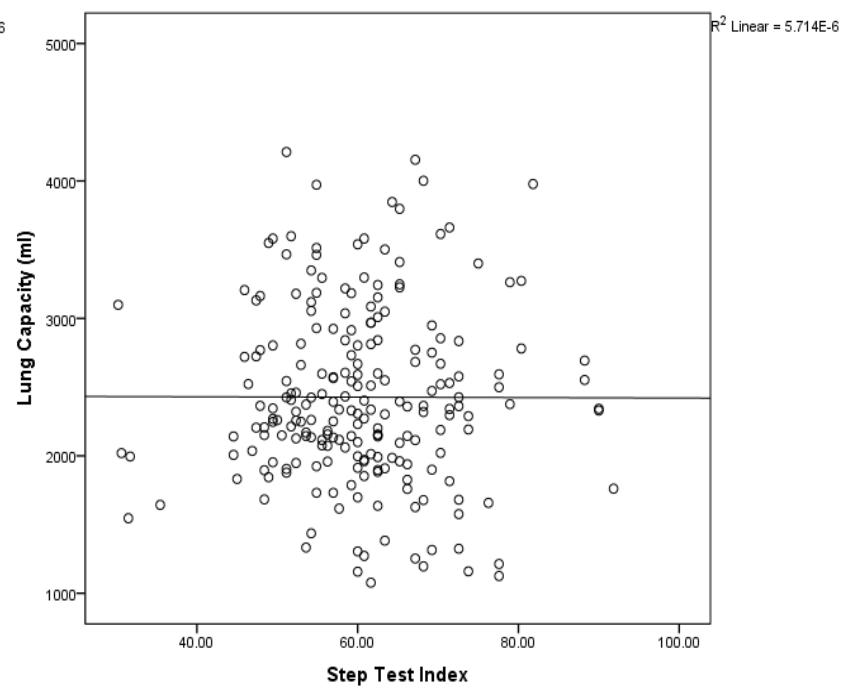

(b) 11-year-old boys

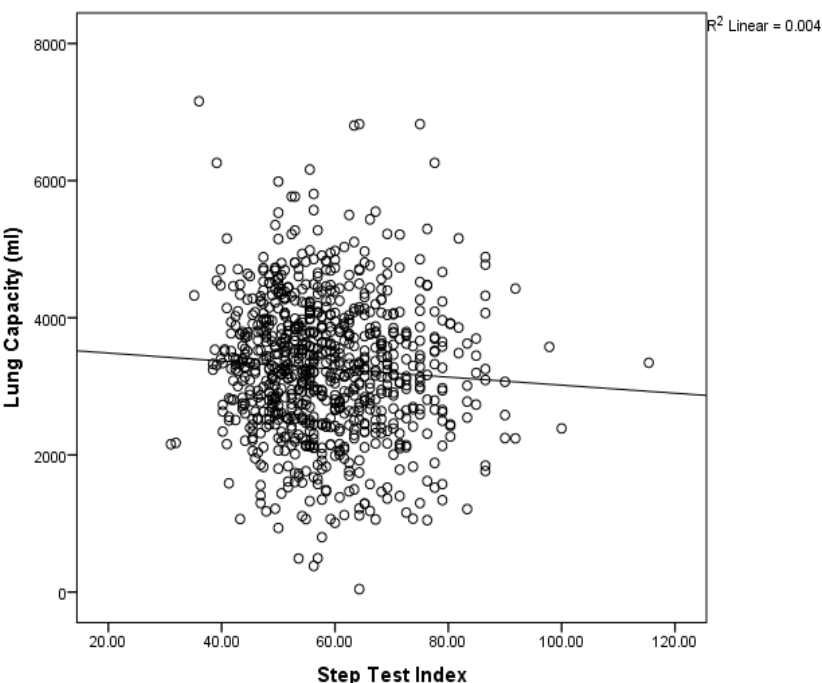

(d) 13-year-old boys

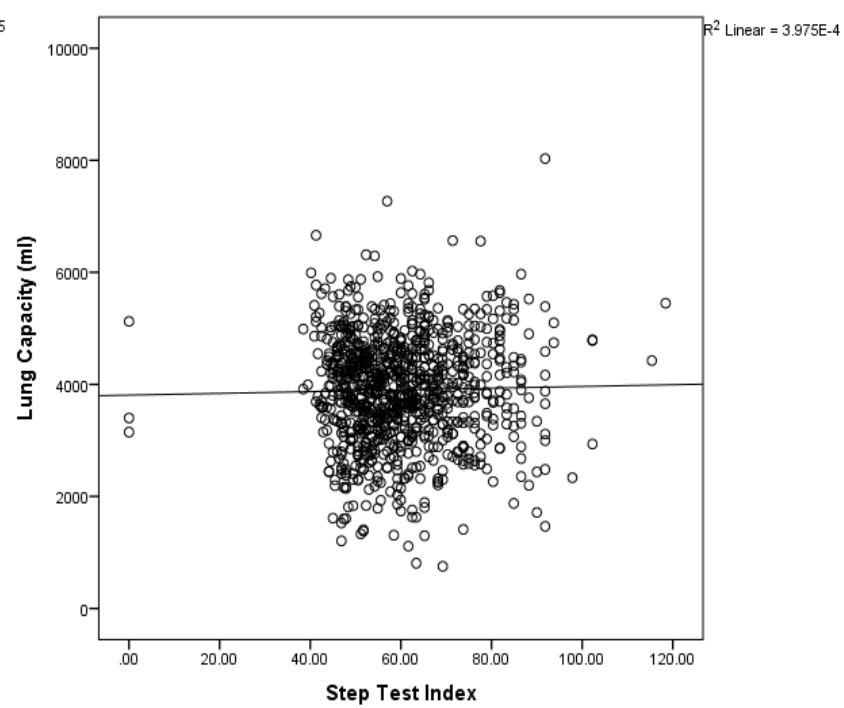

(f) 15-year-old boys 


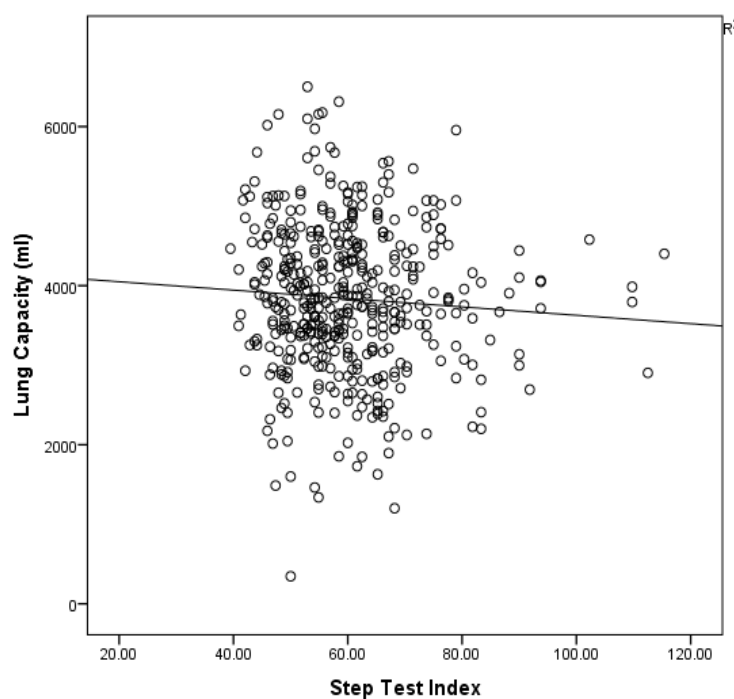

(g) 16-year-old boys

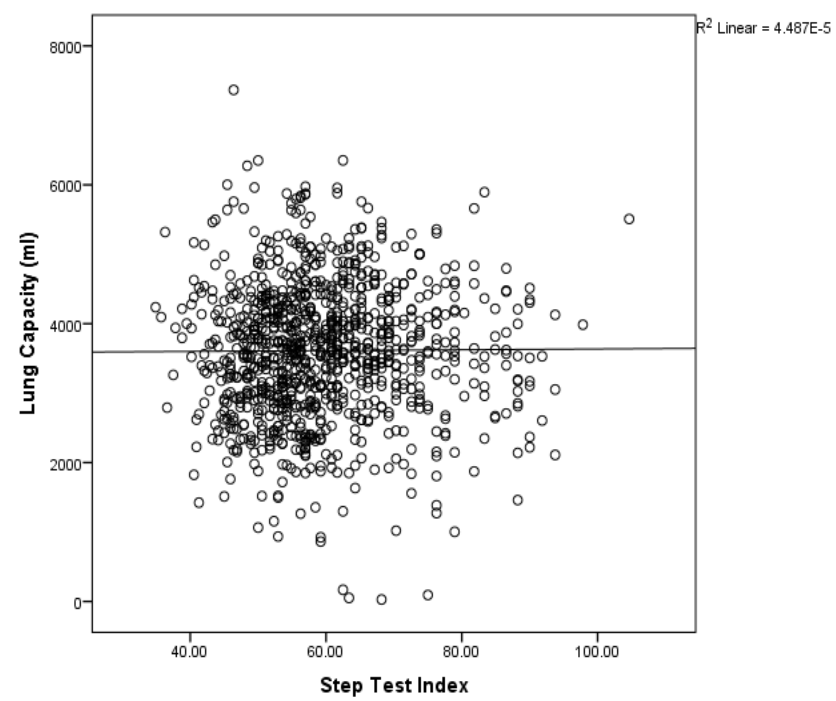

(i) 14-year-old boys

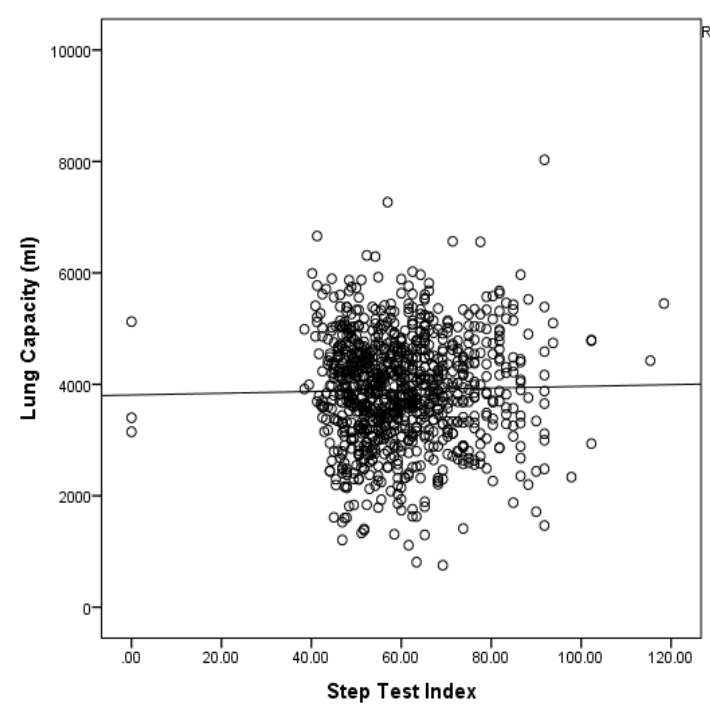

(k) 15-year-old boys

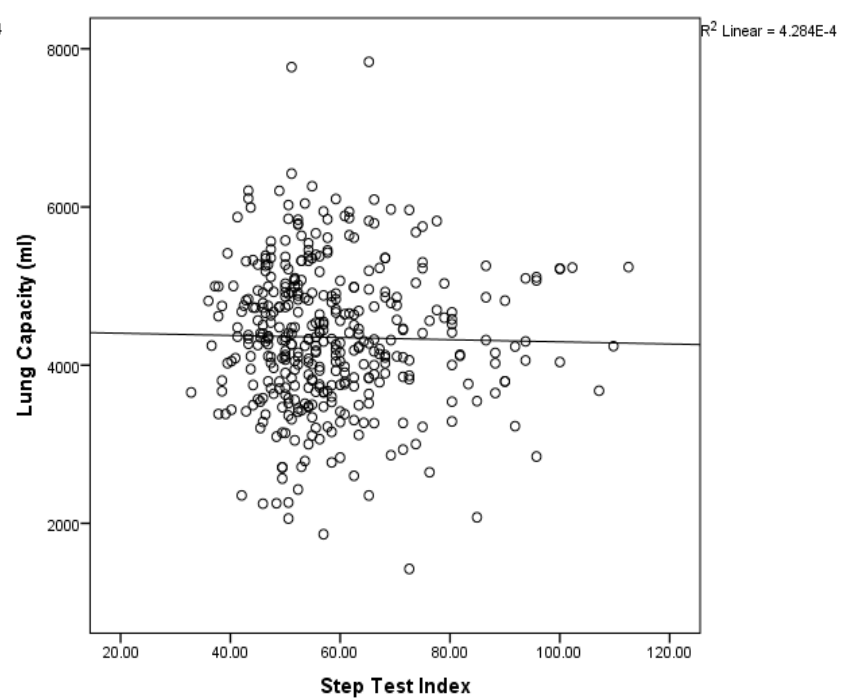

(h) 17-year-old boys

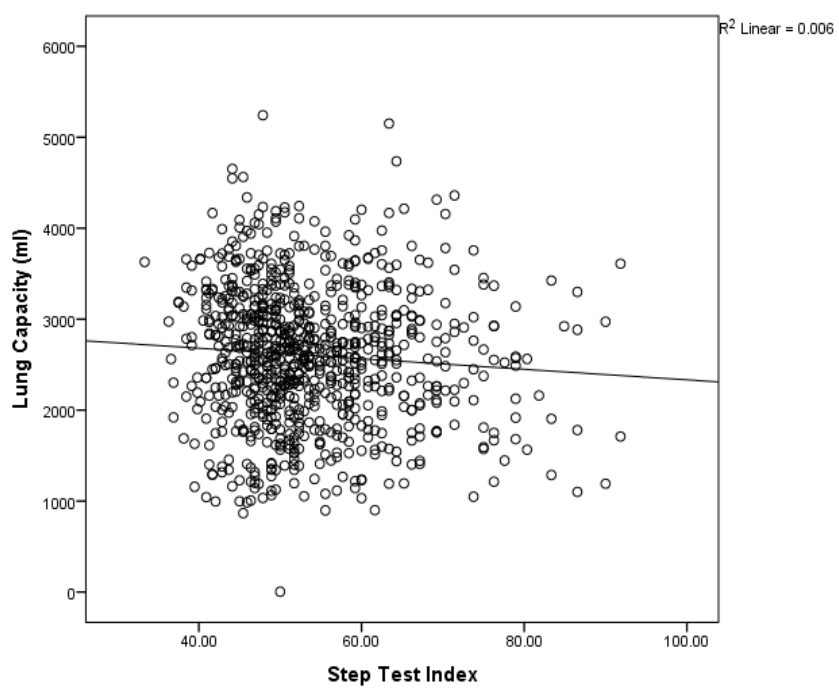

(j) 14-year-old girls

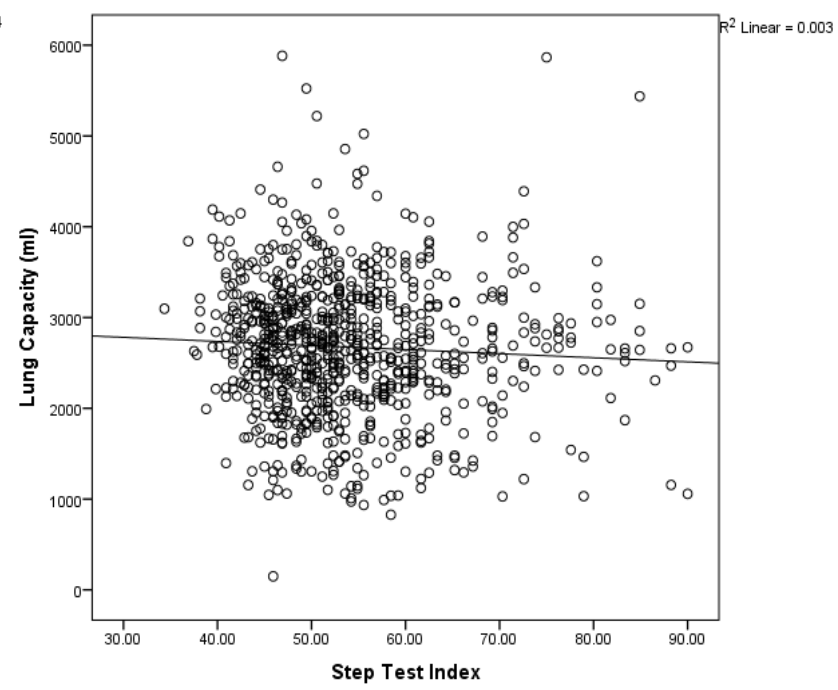

(l) 15-year-old girls 


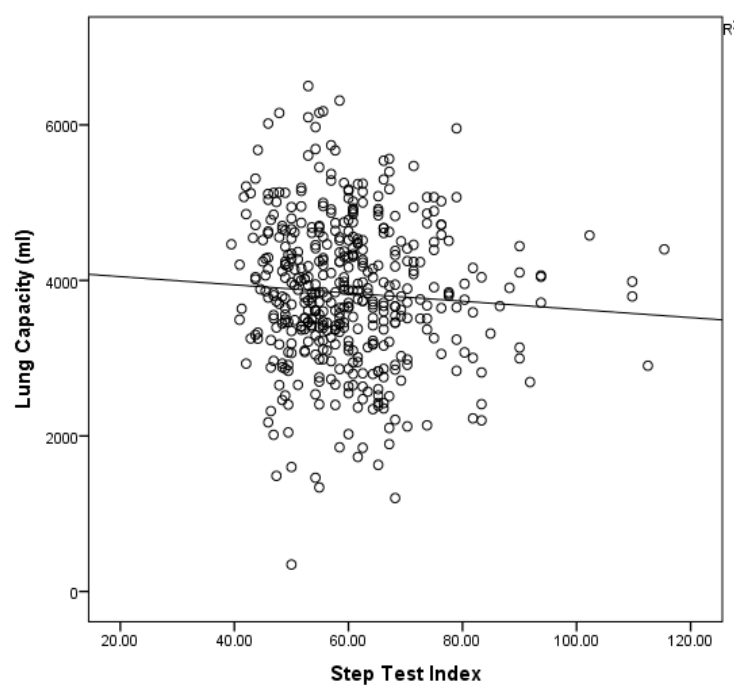

(m) 16-year-old boys

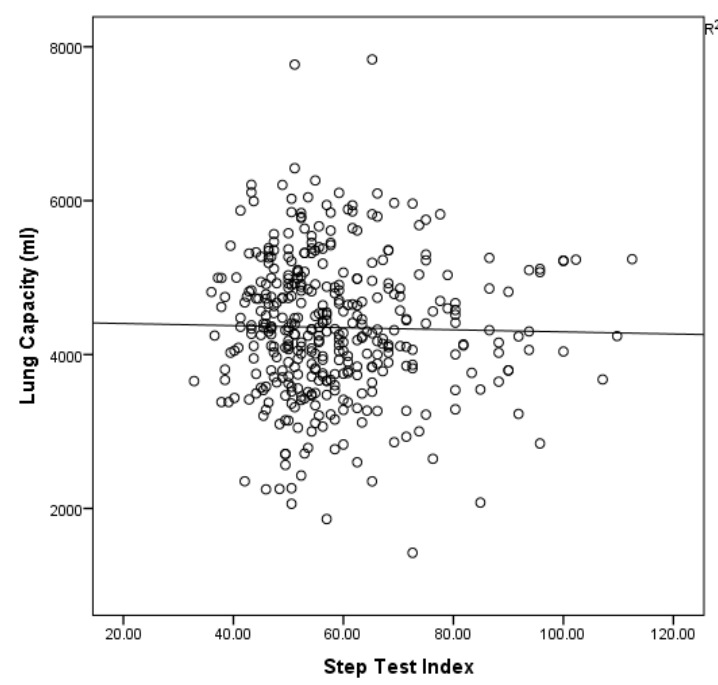

(o) 17-year-old boys

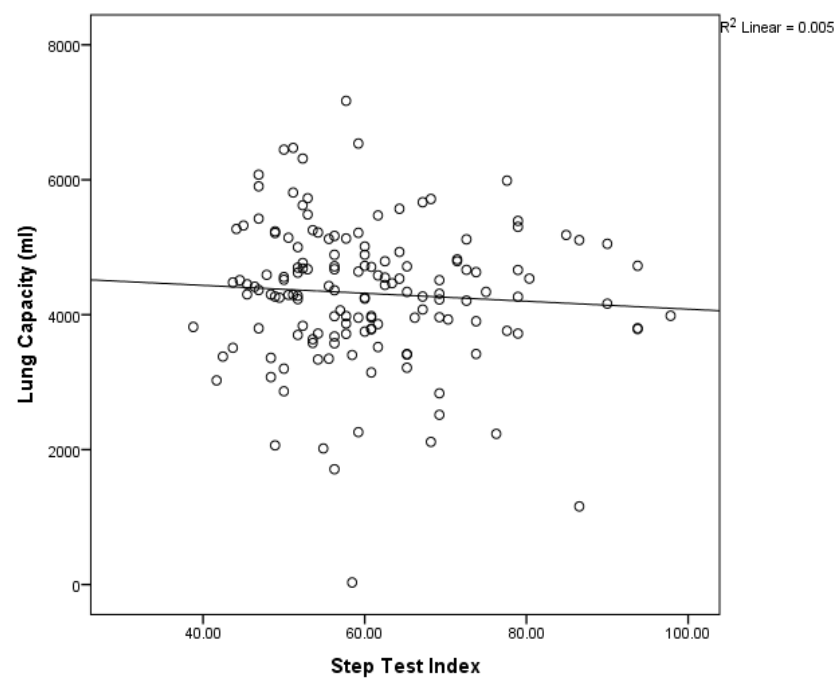

(q) 18-year-old boys

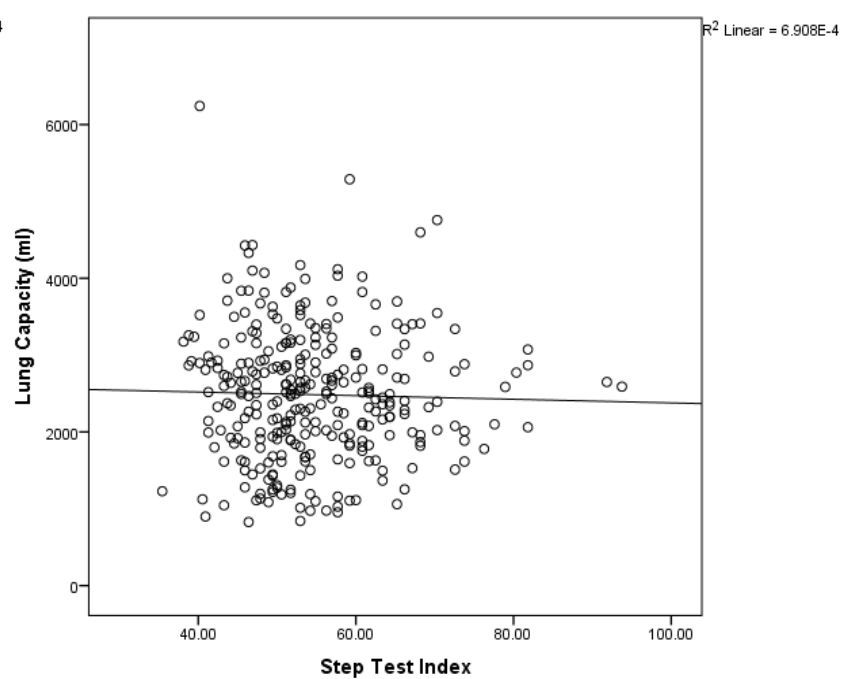

(n) 16-year-old girls

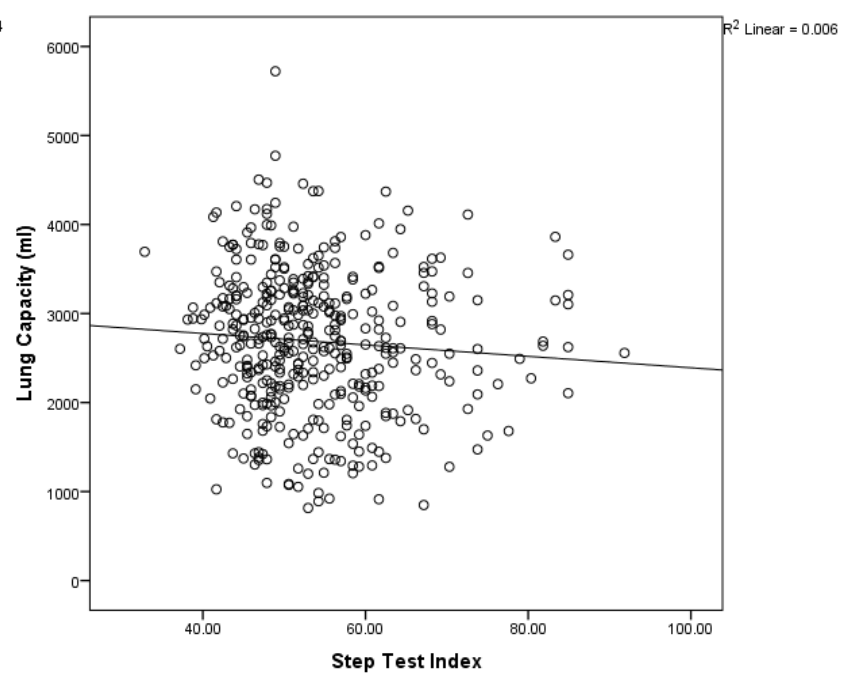

(p) 17-year-old girls

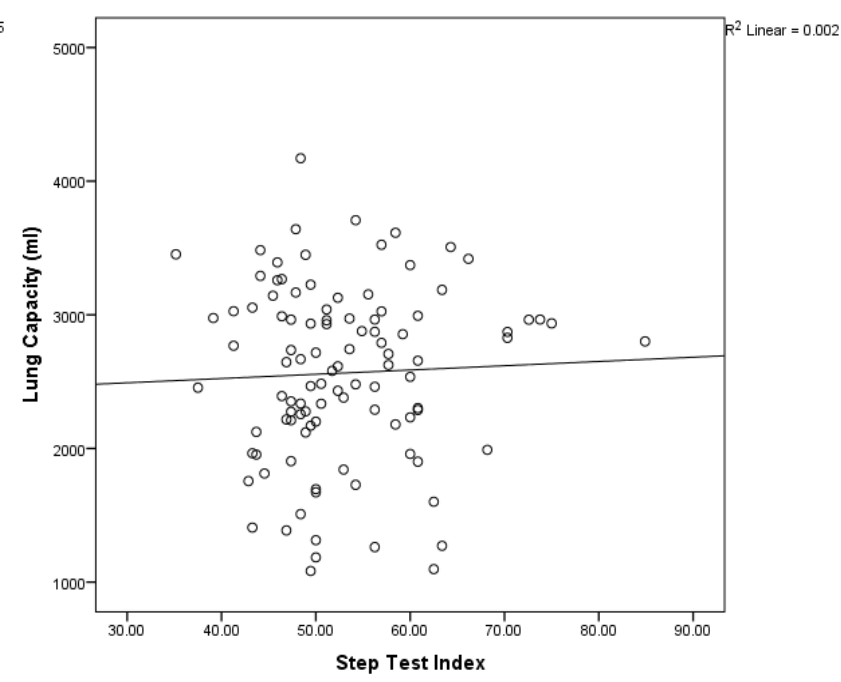

(r) 18-year-old girls

Figure 1. Scatterplots of lung capacity and step test index for boys. 


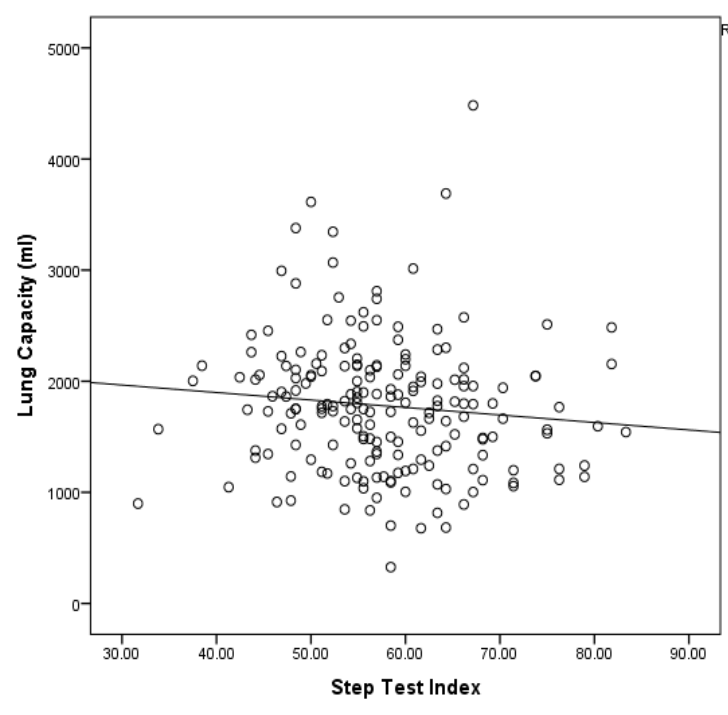

(a) 10-year-old girls

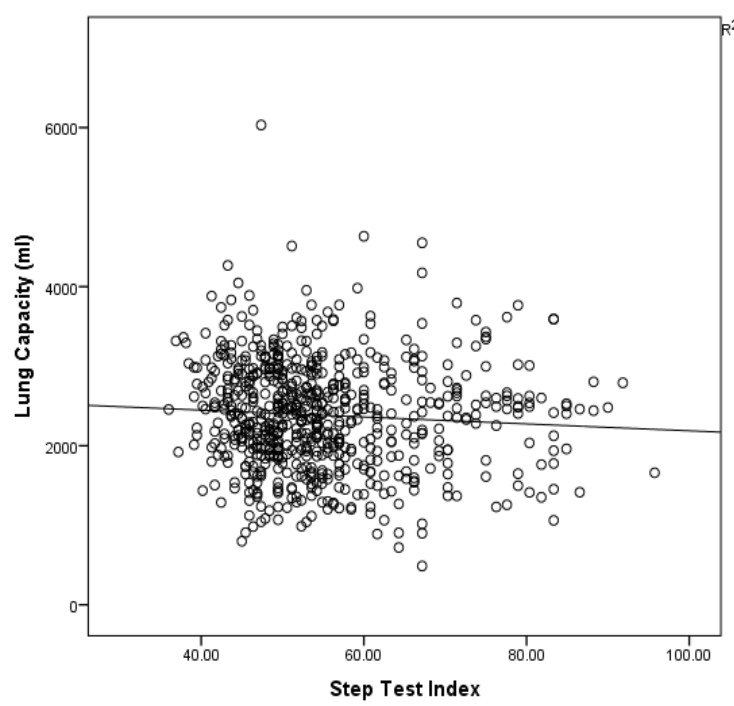

(c) 12-year-old girls

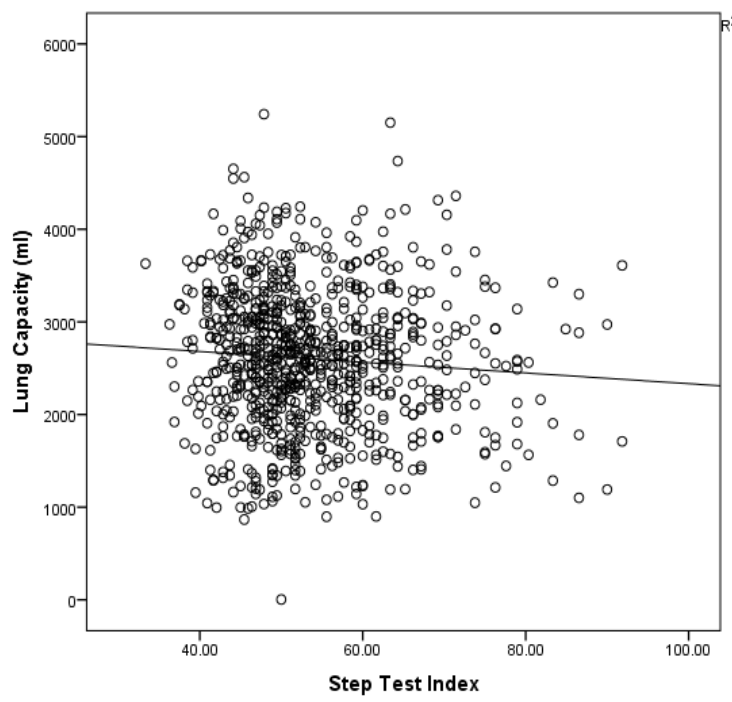

(e) 14-year-old girls

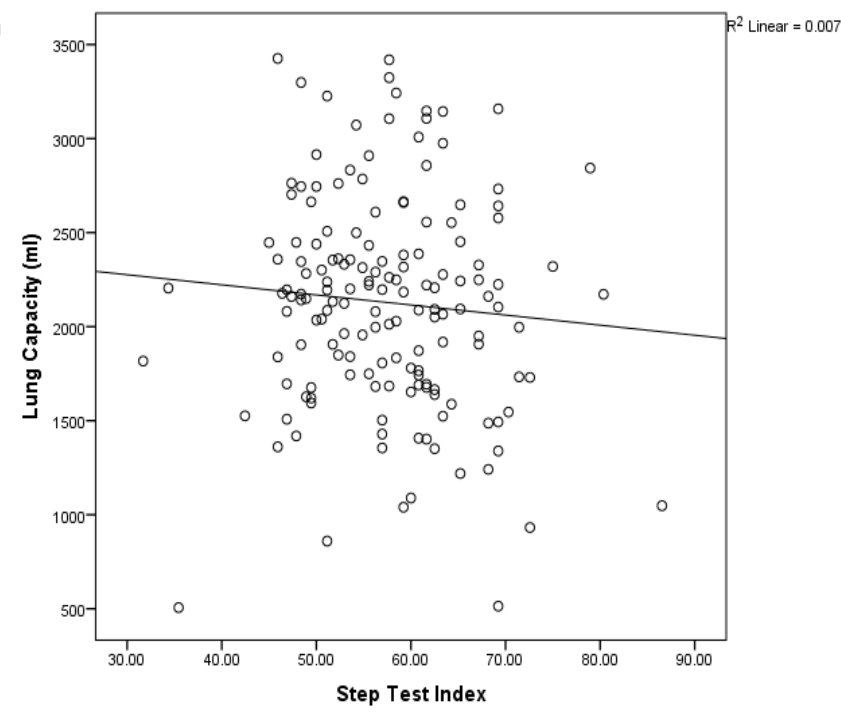

(b) 11-year-old girls

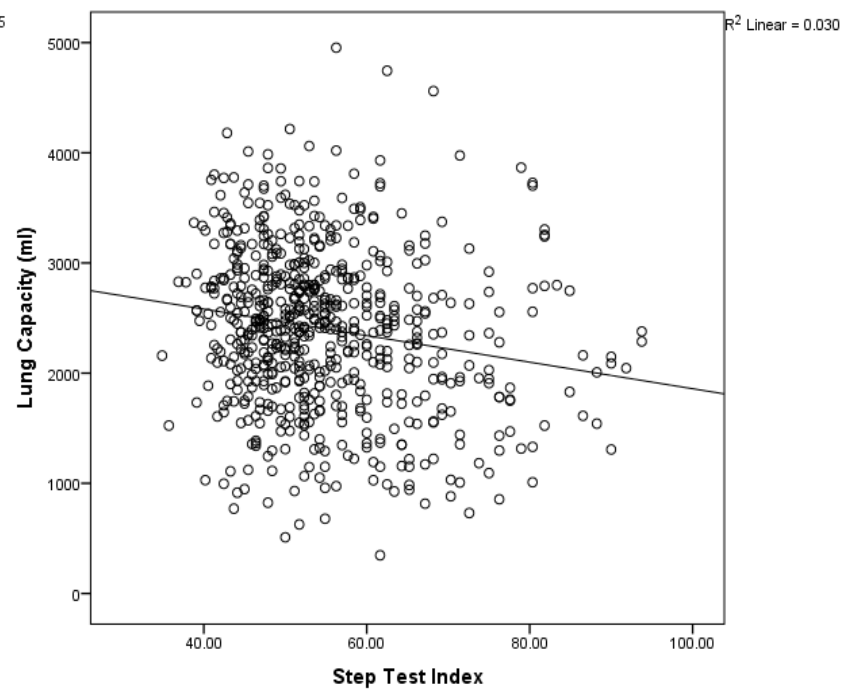

(d) 13-year-old girls

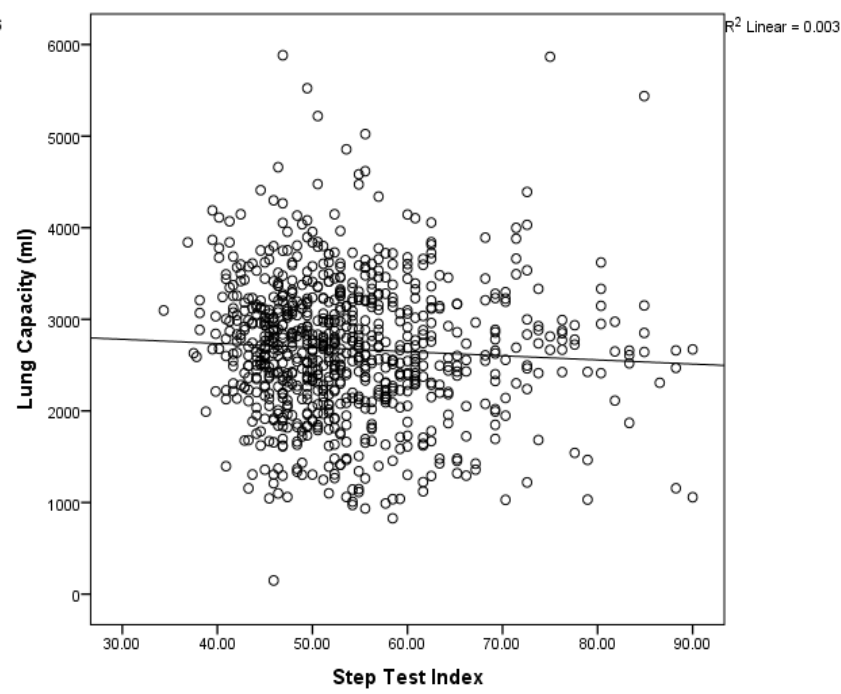

(f) 15-year-old girls 


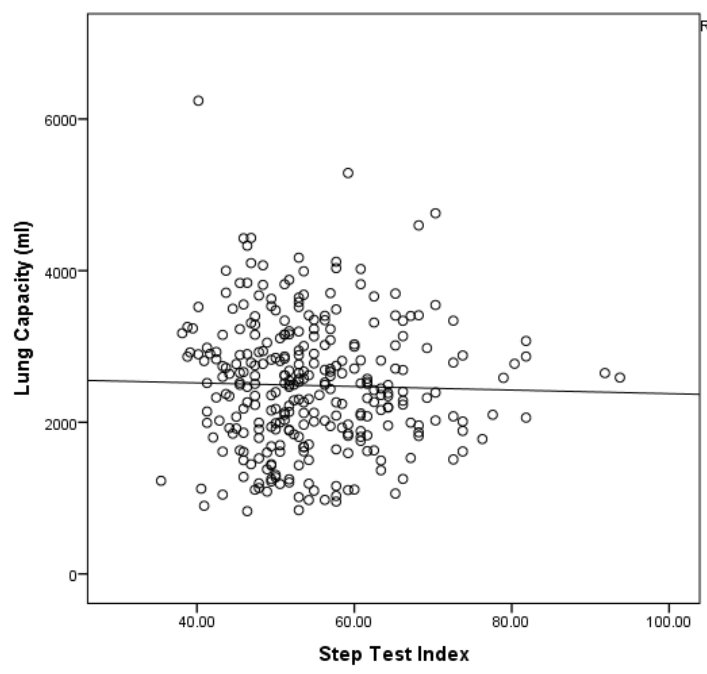

(g) 16-year-old girls

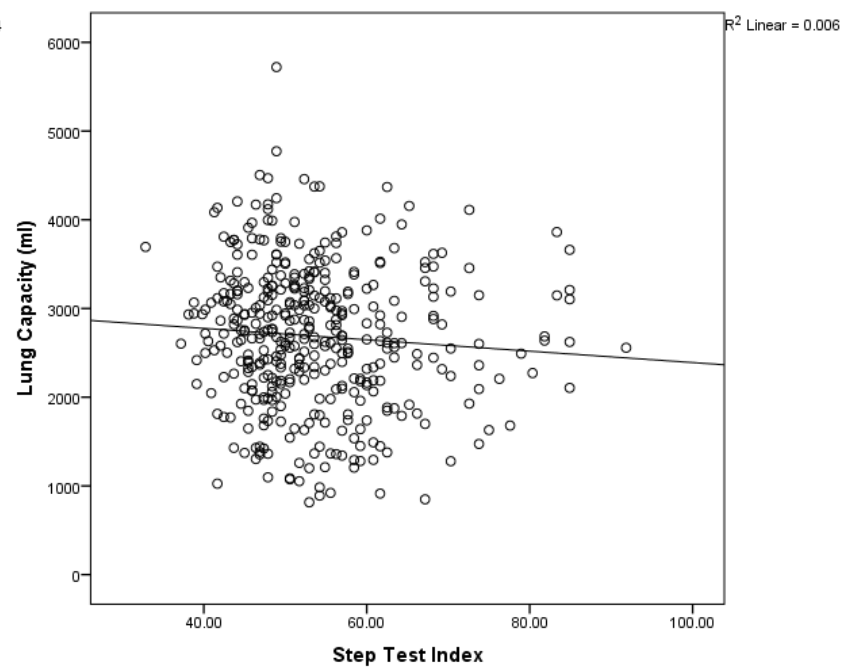

(h) 17-year-old girls

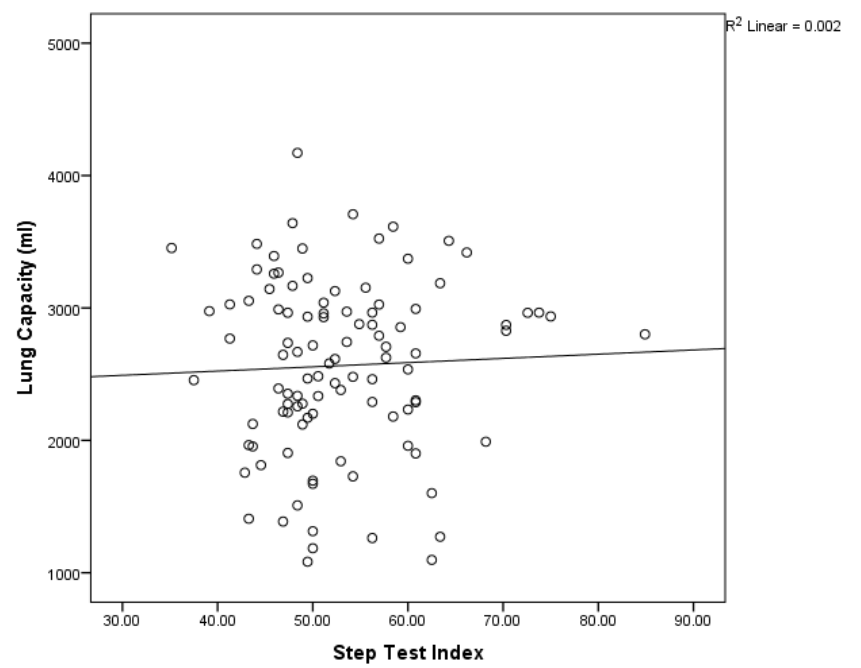

(i) 18-year-old girls

Figure 2. Scatterplots of lung capacity and step test index for girls.

\section{DISCUSSION}

Awareness of the importance of physical fitness is on an upward trend, with a growing body of health-related evidence on muscular capacity, speed and agility. Bouchard, Blair and Haskell [22] classified the health-related components of fitness as cardiorespiratory, musculoskeletal, morphological, and motor and the cardiorespiratory components as submaximal exercise capacity, maximal aerobic power, heart and lung functions and blood pressure. It is clear that although heart and lung functions represent the cardiorespiratory component of fitness, cardiorespiratory testing might produce more accurate results if separated into a cardiovascular function test and pulmonary function test. In the study reported herein, the research team hypothesised that there is a correlation between cardiovascular fitness and pulmonary fitness and tested the hypothesis using a dataset collected from schoolchildren aged 10 to 18 . The advantages of this secondary analysis were the large sample size and its representativeness as a population-based assessment. In addition, the protocol, which was adopted from the China Sports Bureau, is one of the few to separate cardiopulmonary testing into a cardiovascular test and pulmonary test. It was thus deemed useful for determining whether cardiovascular and pulmonary fitness are correlated.

The lung capacity test results show pulmonary capacity to increase with age in both boys and girls, although there are significantly greater variations in this component of fitness as shown in standard deviations for boys between the ages of 13 and 18 . As children are promoted to secondary school at the age of 13 , these findings could possibly due to secondary school life that affect some boys' pulmonary fitness, but not in girls. Further research is necessary to better understand the association between 
school curriculum, adolescent health and fitness. The step test results show this parameter of fitness to remain fairly constant for boys, but to decrease significantly with age for girls. Taking the example of the boys in percentile 50th (P50), the step test index scores were highest at ages $10,11,15,16$ and 18 , with significant drops recorded at ages 12, 13 and 17. Amongst the P50 girls, the step test index scores declined between the ages of 10 and 18 . These profiling results indicate a clear deterioration in pulmonary fitness amongst boys and cardiovascular fitness amongst girls as they grow up. They thus warrant the immediate attention of policymakers and educators, who need to recommend sound physical education or other interventions.

The Pearson correlation coefficients and scatterplots of the lung capacity and step test results provide good evidence of the poor correlation between pulmonary fitness and cardiovascular fitness. However, a correlation test alone cannot give us a clear understanding of whether children who obtain good lung capacity test results will also do well in a step test. In this study, cluster analysis allowed the participating children to be categorised into three clusters with similar characteristics for further verification. The three clusters demonstrated a good degree of similarity amongst the children with excellent results for both test items and a moderate degree of similarity amongst those with good results for both. However, there was less similarity amongst those obtaining a pass or fair result for both test items, which suggests that cardiovascular fitness does not necessarily equate to pulmonary fitness. Further research with stringent controls is required to produce more evidence in support of such a conclusion. The findings reported herein provide a good foundation for research on the comparative efficacy of single tests versus separate tests in evaluating cardiopulmonary fitness.

\section{CONCLUSION}

This study is the first to investigate whether a single field testing parameter can sufficiently reflect cardiopulmonary fitness in a physical fitness assessment. Its findings show that if cardiopulmonary fitness is assessed separately as cardiovascular fitness and pulmonary fitness, there is a poor correlation between the two. Children who obtain good results in cardiovascular functioning may not achieve good results in lung capacity. Stringent maximal tests should be performed in future studies.

\section{REFERENCES}

[1] Mesa, J.L., Ruiz, J.R., Ortega, F.B., Wärnberg, J., González-Lamuño, D., Moreno, L.A., Gutiérrez, A. and Castillo, M.J. (2006) Aerobic physical fitness in relation to blood lipids and fasting glycaemia in adolescents: Influ- ence of weight status. Nutrition, Metabolism \& Cardiovascular Diseases, 16, 285-293.

http://dx.doi.org/10.1016/j.numecd.2006.02.003

[2] Ortega, F.B., Ruiz, J.R., Castillo, M.J. and Sjöström, M. (2008) Physical fitness in childhood and adolescence: A powerful marker of health. International Journal of $\mathrm{Ob}$ esity, 32, 1-11. http://dx.doi.org/10.1038/sj.ijo.0803774

[3] Ruiz, J.R., Castro-Piñero, J., Artero, E.G., Ortega, F.B., Sjöström, M., Suni, J. and Castillo, M.J. (2009) Predictive validity of health-related fitness in youth: A systematic review. British Journal of Sports Medicine, 43, 909-923. http://dx.doi.org/10.1136/bjsm.2008.056499

[4] American College of Sports Medicine (2010) ACSM's health-related physical fitness assessment manual. Lippincott Williams \& Wilkins, Philadelphia.

[5] Andreasi, V., Michelin, E., Rinaldi, A.E.M. and Burini, R.C. (2010) Physical fitness with anthropometric measurements in 7 to 15-year-old school children. Journal of Pediatrics, 86, 497-502.

[6] Castro-Pinero, J., Padilla-Moledo, C., Ortega, F.B., Moliner-Urdiales, D., Keating, X. and Ruiz, J.R. (2012) Cardiorespiratory fitness and fatness are associated with health complaints and health risk behaviors in youth. Journal of Physical Activity \& Health, 9, 642-649.

[7] Okely, A.D., Hardy, L.L., Booth, M.L., Dobbins, T.A., Denney-Wilson, E.A. and Yang, B. (2010) Changes in cardiorespiratory fitness among children and adolescents in Australia: 1997 and 2004. Journal of Sports Science, 28, 851-857. http://dx.doi.org/10.1080/02640411003716959

[8] Ortega, F.B., Artero, E.G., Ruiz, J.R., España-Romero, V., Jiménez-Pavón, D., Vicente-Rodriguez, G. and Castillo, M.J. (2011) Physical fitness levels among European adolescents: The HELENA study. British Journal of Sports Medicine, 45, 20-29.

http://dx.doi.org/10.1136/bjsm.2009.062679

[9] Ruiz, J.R., Ortega, F.B., Gutierrez, A., Meusel, D., Sjöström, M. and Castillo, M.J. (2006) Health-related fitness assessment in childhood and adolescence: A European approach based on the AVENA, EYHS and HELENA studies. Journal of Public Health, 14, 269-277. http://dx.doi.org/10.1007/s10389-006-0059-z

[10] Tomkinson, G.R., Leger, L.A., Olds, T.S. and Cazorla, G. (2003) Secular trends in the performance of children and adolescents (1980-2000): An analysis of 55 studies of the $20 \mathrm{~m}$ shuttle run test in 11 countries. Sports Medicine, 33, 285-300. http://dx.doi.org/10.2165/00007256-200333040-00003

[11] Stratton, G., Canoy, D., Boddy, L.M., Taylor, S.R., Hackett, A.F. and Buchan, I.E. (2007) Cardiorespiratory fitness and body mass index of 9- 11-year-old English children: A serial cross-sectional study from 1998 to 2004. International Journal of Obesity, 31, 1172-1178. http://dx.doi.org/10.1038/sj.ijo.0803562

[12] Jacks, D.E., Topp, R. and Moore, J.B. (2011) Prediction of VO2 peak using a sub-maximal bench step test in children. Clinical Kinesiology, 65, 68-75.

[13] Montoye, H.J. (1953) The Harvard step test and work capacity. Reviews of Canadian Biology, 11, 491-499. 
[14] Golding, L. (2000) YMCA fitness testing and assessment manual, 4th Edition, Human Kinetics, Champaign.

[15] Chief Secretary for Sport of General Administration of Sport of China (2002) Annual report of national constitution assessment in 2000. Beijing University of Physical Education Press, Beijing.

[16] Borel, B., Fabre, C., Saison, S., Bart, F. and Grosbois, J.M. (2010) An original field evaluation test for chronic obstructive pulmonary disease population: The six-minute stepper test. Clinical Rehabilitation, 24, 82-93. http://dx.doi.org/10.1177/0269215509343848

[17] Klein, O.L., Krishnan, J.A., Glick, S. and Smith, L.J. (2010) Systematic review of the association between lung function and Type 2 diabetes mellitus. Diabetic Medicine, 27, 977-987. http://dx.doi.org/10.1111/j.1464-5491.2010.03073.x

[18] Bongers, B.C., Hulzebos, E.H.J., Arets, B.G.M. and Takken, T. (2012) Validity of the oxygen uptake efficiency slope in children with cystic fibrosis and mild-to- moderate airflow obstruction. Pediatric Exercise Science, 24,
129-141.

[19] Chung, L.M.Y., Chung, J.W.Y. and Wong, T.K.S. (2012) Relationship between physical fitness and aging among the older Chinese. Asian Journal of Gerontology \& Geriatrics, 7, 69-79.

[20] Chung, J.W.Y., Chung, L.M.Y., Wong, F.H.T. and Chen, B. (2009) The impact of lifestyle on the physical fitness of primary school children. Journal of Clinical Nursing, 18, 1002-1009. http://dx.doi.org/10.1111/j.1365-2702.2008.02420.x

[21] Chung, L.M.Y., Chow, L.P.Y. and Chung, J.W.Y. (2013) Normative reference of standing long jump indicates gender difference in lower muscular strength of pubertal growth. Health, 5, 6-11. http://dx.doi.org/10.4236/health.2013.56A3002

[22] Bouchard, C., Blair, S.N. and Haskell, W.L. (2007) Why study physical activity and health? In: Bouchard, C., Blair, S.N. and Haskell, W.L., Eds., Physical Activity and Health, Human Kinetics Books, Champaign, 3-22. 